\title{
THE EFFECT OF BASELINE LUNG FUNCTION ON THE DETERMINATION OF TIME TO BRONCHIOLITIS OBLITERANS SYNDROME
}

Christopher M. Burton, MD*, Martin Iversen, MD, PhD*, Jørn Carlsen, MD, PhD*, Claus Andersen, MD, PhD* and Jann Mortensen, MD, PhD'

Lung Transplantation Best Abstract Award, sponsored by Vitrolife Sweden

*Dept of Cardiology, Division of Lung Transplantation

\#Dept of Pathology

'Dept of Nuclear Medicine \& Clinical Physiology, Copenhagen University Hospital, Rigshospitalet, Copenhagen, Denmark

WINNING ABSTRACT: Long term survival after lung transplantation depends on the development and severity of bronchiolitis obliterans syndrome (BOS).

The objective of this study was to identify the relationship between baseline FEV 1 and transplant procedure type, on the development and progression of BOS grade Op to 3. All patients receiving a cadaveric lung transplant 1992-2004 were included in the study $(n=389)$. Exclusion criteria were patients surviving $<3$ months $(n=39)$ and missing spirometry measurements $(n=4)$.

There were significant differences between the transplant procedures, heart-lung (HLTx), double-lung (DLTx), and singlelung (SLTx), with respect to recipient age, BMI, and indication for transplantation. Baseline FEV 1 for HLTx (median $2.9 \mathrm{~L}$, quartiles 2.3-4.3L) and DLTx recipients (median 2.9L, quartiles 2.4-3.7L) were significantly larger than for patients undergoing SLTx procedures (median 1.6L, quartiles 1.3-1.9L), $p<0.0001$, respectively. Survival analyses demonstrated a significant association between baseline FEV1 per litre and the development of BOS: grade Op (HR: 0.59, CI: $0.51-0.68, p<0.0001$ ); grade 1 (HR: 0.60, Cl: 0.51-0.70. p<0.0001); grade 2 (HR: 0.62, Cl 0.52-0.74, p<0.0001); and grade 3 (HR: 0.73, Cl 0.60-0.89, p=0.002). There was a significant log-log linear relationship between baseline FEV1 and time to the development of BOS grades $0 p$ to 3 in all patients developing the respective $\mathrm{BOS}$ grade $(\mathrm{p}<0.0001$, respectively).

Baseline lung function is an important confounder and should be considered in future risk factor evaluations for the development and progression to BOS.

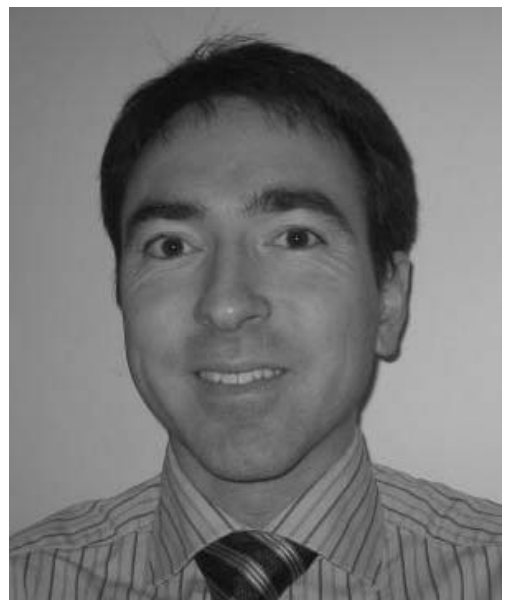

Christopher M. Burton

Dept of Cardiology, Division of Lung Transplantation, Rigshospitalet, Copenhagen, Denmark

\section{SYNOPSIS OF MY JOB AND THE ROLE OF THE UNIT IN WHICH I WORK}

Since 2003, I have worked as a pulmonary medicine research fellow at the Division of Lung Transplantation (Copenhagen University Hospital, Rigshospitalet, Copenhagen, Denmark).

STATEMENT OF INTEREST: None declared.
This is the only centre to perform lung transplantation in Denmark. Since the start of the programme in 1992 the centre has carried out $\sim 500$ lung transplants on recipients with survival rate superior to the international registry data, and comparable with other published large volume centres. I was originally employed to collect core pre-transplant, operative and post-transplant information on all transplanted patients from this centre, as part of an initiative to share data with the other Scandinavian heart and lung transplant centres. This exchange of data is governed by the Nordic Thoracic Transplant Study Group and is hosted by Scandiatransplant, the Scandinavian transplant registry [2]. The standardised collection of such a large number of variables will be a reliable source of data for ongoing multicentre retrospective and prospective studies [3].

This task led to the design and implementation of a clinical database that, due to its versatility, has been employed to store a wealth of additional data, and has been easily adapted to coordinate a number of other research initiatives. The database is also being used to collect data prospectively, and forms the basis of several PhD projects, including my own which I began in January 2005 and is sponsored by the University of Copenhagen.

\section{SYNOPSIS OF MY RESEARCH AND HOW MY WINNING POSTER IS PART OF THIS}

Lung transplantation is an accepted treatment for patients with end-stage lung disease who are unresponsive to alternative 
medical or surgical interventions. However, survival after lung transplantation remains poor relative to other forms of solid organ transplantation. One of the principal reasons for this is the development of chronic allograft dysfunction [4]. The histological appearance of bronchiolitis obliterans (BO) was originally proposed as a marker of chronic rejection owing to the morphological similarities between $\mathrm{BO}$ and lesions identified in other solid organ allografts. As BO was difficult to diagnose by transbronchial biopsy, a surrogate marker of $\mathrm{BO}$, termed bronchiolitis obliterans syndrome (BOS), was proposed as a measure of chronic allograft dysfunction [5]. BOS is defined and graded according to a sustained decline in forced expiratory volume in one second (FEV1) relative to a maximal post-transplant baseline.

The objectives of my ongoing $\mathrm{PhD}$, entitled "Inflammatory lesions in the pulmonary allograft", are to evaluate whether interstitial inflammatory lesions other than acute cellular rejection: 1) herald the development of clinical BOS and/or histological $\mathrm{BO} ; 2$ ) have a deleterious effect on patient survival independent on the development of BOS; and 3) represent new targets for treatment in the field of lung transplantation.

During my analyses of BOS, it occurred to me that since BOS is defined relative to a baseline FEV1, the time to the development and progression of BOS would be dependent on the absolute value of the baseline FEV1. For example, if two patients (A and B) underwent lung transplantation and patient $\mathrm{A}$ had a baseline FEV1 of $4 \mathrm{~L}$ and patient $\mathrm{B}$ a baseline FEV1 of $2 \mathrm{~L}$, then BOS would be present if patient $B$ lost $0.4 \mathrm{~L}$ in FEV1 and if patient $\mathrm{A}$ lost $0.8 \mathrm{~L}$. If both patients were declining in FEV1 at exactly the same rate, patient B would develop BOS before patient A. Since $\mathrm{BOS}$ is a proposed measure of the rate of decline in lung function, the result misleadingly indicates that patient $B$ is doing worse than patient A. The truth is that, for whatever reason, patient $B$ achieved a lower post-transplant baseline than patient A. This may have been the result of the type of lung transplant (single versus double) or donor/recipient sex and age, and not a reflection of allograft dysfunction.

The presented abstract demonstrates the effect of baseline FEV1 on the determination of BOS, and concludes that the absolute value of baseline FEV1 constitutes a systematic bias in population analyses of freedom from BOS.

\section{HOW MY RESEARCH FITS IN WITH THE OVERALL RESEARCH OF MY WORKING GROUP/RESEARCH TEAM}

The observation that baseline FEV1 constitutes a systematic bias in the definition of BOS is very important, and will need to be accounted for in future analyses of BOS, as well as in my ongoing PhD.

As a result of this finding, we now routinely evaluate the effect of variables of interest, not only on the time to BOS adjusting for the confounding of baseline FEV1, but also on the absolute value of baseline FEV1. For instance, reperfusion injury of the pulmonary allograft has been associated with early mortality and late morbidity $[6,7]$. Several authors have also shown an association between the development of reperfusion injury and earlier development of BOS [8,9]. Importantly, these studies have not adjusted for the confounding of baseline FEV1, therefore, the apparently more rapid progression to BOS may have resulted from an association between reperfusion injury and a lower baseline FEV1 [10]. If reperfusion injury is indeed associated with reduced maximal FEV1, but does not alter the rate of decline of lung function thereafter, this lower maximal FEV1 would still be expected to confer a survival disadvantage and limit exercise tolerance.

Distinguishing maximal FEV1 from the rate of decline in FEV1 will be important in understanding the natural history of posttransplant lung function and in accurately identifying patients with more rapid chronic pulmonary allograft dysfunction, so as to assess potential risk factors and determine the effects of therapeutic intervention.

\section{HOW MY RESEARCH WILL IMPACT ON CLINICAL OR RESEARCH PRACTICE}

This work highlights the fact that, despite the apparently simple definition of BOS in relation to a post-transplant baseline, the application and interpretation of BOS grading criteria in large population analyses is complex.

In addition to the aforementioned effects of absolute baseline FEV1 on the time to BOS, a number of other factors should also be considered in analyses of BOS.

Since post-transplant FEV1 is a dynamic measurement, patients who may appear to have a sustained decline in FEV1 (i.e. BOS) at one time may subsequently experience an improvment in FEV1 to a level above the threshold of BOS (or BOS grade of interest). Thus, the accuracy of determining true BOS is likely to increase as the duration of follow-up increases and is only unequivocal when there is complete follow-up, i.e. the patient has died. The accuracy of determining BOS also depends on the frequency of spirometry measurement, the accuracy of the measurement device, and the compliance of the patient. Thus, unless measured daily, BOS can only be determined to within an inter-measurement interval.

In addition to the effects of transplant type on determining baseline FEV1, questions remain as to whether BOS grades can be compared between patients receiving single or bilateral lung transplantation. The contribution of the native lung to total FEV1 in single lung recipients is difficult to estimate and may vary depending on the pre-transplant disease. The native lung is not subject to the same alloimmune rejection processes, and may, therefore, constitute an increasing percentage of the measured FEV1 as lung function declines. In this way, the native lung would buffer the effect of BOS on FEV1.

Furthermore, the development of BOS and death occurring prior to the development of BOS, are competing events. In this situation, straightforward freedom from BOS survival analyses may be misleading. Traditionally, authors have presented data on BOS-free survival as well as freedom from BOS, but since BOS-free survival is a combined end-point of BOS or death, the interpretation of results is difficult. We have increasingly employed competing risk analyses as a supplement to existing freedom from BOS and BOS-free survival analyses, and have found that the competing risk analyses can sometimes provide additional insights into the assessment of risk factors for BOS.

We hope that other centres will continue to suggest refinements to the diagnosis and analysis of bronchiolitis obliterans syndrome. Improving the reliability of bronchiolitis obliterans syndrome reporting is critical in the identification of risk 
factors and therapeutic interventions for this most important limitation to long-term survival after lung transplantation.

\section{REFERENCES}

1 Burton CM, Milman N, Carlsen J, et al. The Copenhagen National Lung Transplant Group: survival after single lung, double lung, and heart-lung transplantation. J Heart Lung Transplant 2005; 24: 1834-1843.

2 The Scandinavian Transplant Society. www.scandiatransplant.org

3 Andersson B. Thoracic organ transplantations in the Nordic countries 1983-2005, Nordic Thoracic Transplantation Study Group. 1st official registry report-2006. J Heart Lung Transplant 2007; 26: Suppl. 1, S175.

4 Trulock EP, Edwards LB, Taylor DO, et al. Registry of the International Society for Heart and Lung Transplantation: twenty-third official adult lung and heart-lung transplantation report-2006. J Heart Lung Transplant 2006; 25: 880-892.

5 Cooper JD, Billingham M, Egan T, et al. A working formulation for the standardization of nomenclature and for clinical staging of chronic dysfunction in lung allografts. International Society for Heart and Lung Transplantation. J Heart Lung Transplant 1993; 12: 713-716.

6 Christie JD, Sager JS, Kimmel SE, et al. Impact of primary graft failure on outcomes following lung transplantation. Chest 2005; 127: 161-165.

7 Arcasoy SM, Fisher A, Hachem RR, Scavuzzo M, Ware LB, ISHLT Working Group on Primary Lung Graft Dysfunction. Report of the ISHLT Working Group on Primary Lung Graft Dysfunction part V: predictors and outcomes. J Heart Lung Transplant 2005; 24: 1483-1488.

8 Daud SA, Yusen RD, Meyers BF, et al. Impact of immediate primary lung allograft dysfunction on bronchiolitis obliterans syndrome. Am J Respir Crit Care Med 2007; 175: 507-513.

9 Fiser SM, Tribble CG, Long SM, et al. Ischemia-reperfusion injury after lung transplantation increases risk of late bronchiolitis obliterans syndrome. Ann Thorac Surg 2002; 73: 1041-1047.

10 Burton CM, Iversen M, Milman N, et al. Outcome of lung transplanted patients with primary graft dysfunction. Eur J Cardiothorac Surg 2007; 31: 75-82. 